\title{
Development of Automated Glass Frosting Machine
}

\author{
Apurva D. Joshi \\ Department Mechanical \\ Engineering, \\ Yashwantrao Chavan College \\ of Engineering, Rashtrasant \\ Tukadoji Maharaj Nagpur \\ University, Nagpur (M.S.) - \\ India.
}

\author{
D.I Sangotra \\ Department Mechanical \\ Engineering, \\ Yashwantrao Chavan College \\ of Engineering, Rashtrasant \\ Tukadoji Maharaj Nagpur \\ University, Nagpur (M.S.) - \\ India.
}

\author{
S.T Bagde \\ Department Mechanical \\ Engineering \\ Yashwantrao Chavan College \\ of Engineering, Rashtrasant \\ Tukadoji Maharaj Nagpur \\ University, Nagpur (M.S.) - \\ India.
}

\begin{abstract}
As Industry is turning more and more to computer-based automation; very complicated and precise components having some special requirements are demanded by these industries. The demand is also increasing for glass industry; they are still produced on handicraft scale essentially involving manual labor. To avoid child labor and the manual process of sandblasting is very hazardous and creates dust, therefore there is need to automate the Glass frosting technique, so we are proposing the Automated Glass frosting machine. Glass frosting is one of the etching methods of imprinting images on glass. Glass frosting involves creating a design on a glass surface by applying an acidic or abrasive substance. In a proposed concept the glass is stationary and sand is filled in nozzle and sand blasting will be done with the help of nozzle and the nozzle will move accordingly to carve a stencil on glass. With the help of Solid works the assembly is made and interfacing is done by using the KIEL software and flash magic.
\end{abstract}

Keywords: Glass etching, Sandblasting, Acid etching, and Chemical etching.

\section{INTRODUCTION}

With the pressing need for increased productivity and delivery of end products of uniform Quality, industry is turning more and more to computer-based automation. At the present time, most of industrial automated manufacturing is carried out by special purpose machines, designed to perform specific functions in a manufacturing process. The conventional methods, in spite of recent advancements are inadequate to machine such materials from stand point of accuracy, precision and economic production. At the present time, most of industrial automated manufacturing is carried out by special purpose machines, designed to perform specific functions in a manufacturing process.

Glass Frosting is an age-old old method of imprinting images on glass. There are three ways to create a piece of etched the glass.
1. Acid-etching
2. Chemical-etching
3. Sand-blasting

\subsubsection{Acid etching:}

Acid etching uses an acid resistant material to cover areas of the glass that the artist wants protected. Hydrofluoric acid is then applied to the glass to produce the design.

\subsubsection{Chemical etching:}

Chemical etching is another way to produce etched glass and is normally what is found in glass etching kits. Just as in sandblasting, a stencil is used to protect the glass where the etching effect is not desired. Instead of an abrasive, however, a chemical cream is applied to the glass.

Acid etching can create the same appearance as sandblasted glass. One of the major advantages of acid etching over sand blasting is that it can be done simply and without as many tools. A frosting effect can also be achieved using different strengths of acid etching compounds.

\subsubsection{Sandblasting}

Sandblasting, also known as abrasive blasting is a process by which small particles of sand or another hard crystalline material are "blasted" through a small funnel or hose using pressurized water or air. The process is commonly used to strip away old paint, to remove rust, to clean stone buildings or to decorate glass. The manual activity of etching process could be subjective and highly dependent on the experience of workers. So glass etching techniques are being increasingly used to save the time.

Sand blasting is an old technique. It was and is used for a wide variety of purposes like tombstone engraving, paint or corrosion removal, house facades cleaning, and surface roughening for better adhesion, device demarking, and glass decoration and (live) tooth drilling. Since the Second World War, sand blasting is used to 
systematically investigate the erosion resistance of materials. This field of research was important for e.g. gas turbines and pipelines and has grown very large. This provides us with a large amount of knowledge on equipment and particle-target interaction. The development of flat panel displays in the nineties caused sand blasting to be developed to an accurate machining method.

Sandblasting allows for greater variation through the use of different degrees of coarseness in sand, and also for depth blasting, giving the finished product a rich textured appearance. Sand blasting is a very interesting technology; the sandblasting glass etching process consists of corroding glass by violently projecting sand upon its surface by means of a current of air or steam. The tube conveying the current of air or steam terminates at a nozzle containing a series of fine holes. The sand is thrown violently against the glass plate or any other body placed within its range, and thus exerts a corroding action. By varying the quantity of the sand, the volume and velocity of the current, as well as the diameter of the jet, the desired effects are obtained.

\section{LITERATURE SURVEY}

1.The manual activity of etching process could be subjective and highly dependent on the experience of workers. So glass etching techniques are being increasingly used to save the time.

2. Sand blasting is an old technique. It was and is used for a wide variety of purposes like tombstone engraving, paint or corrosion removal, house facades cleaning, and surface roughening for better adhesion, device demarking, and glass decoration and (live) tooth drilling. Powder blasting is very interesting technology for the realization of 3 dimensional microstructures in brittle materials like glass or silicon. In this paper detail basics of oblique powder blasting techniques process, by varying the angle the angle of powder beam \& using mathematical procedure it will able to separate the erosion effects of primary impacting particles \& will create new options for 3 dimensional micro fabrication of brittle materials. $[1,2]$
1. From the above paper [1]; realize with its complex 3dimensional \& monolithic suspended microstructures in glass. Comparison has been done with metal contact mask \& alternative is found photosensitive flex polymer. [3]

2. The general information on particle target interaction and powder blasting conditions are explained. Also discusses some of these mask types. The surface roughness created by powder blasting is much higher compared to other micromachining methods. Therefore this thesis shows how the roughness is created and how it can be manipulated. To further decrease the minimum feature size, the average particle size also has to be decreased. However, the removal rate drops sharply if the particles become too small, a detailed description of this transition, the typical channel profiles that are created by powder blasting are discussed. Effects can be reduced by changing the particle size or the jet impact. [4]

3. Abrasive particles are propelled by an inert gas of velocity. When directed at a work piece, resulting erosion can be used for cutting, drilling, etching \& polishing. The paper testing \& analyze various process parameters of abrasive jet machining is explained in detail. [5]

4. Mechanism of AJM, how AJM works, study of process parameters are explained in detail. Various observations have been seen and from the observation what range should we select for process is explained. As Nozzle Tip Distance increases, the Top surface diameter and Bottom surface diameter increases. As the Pressure increases Material Removal Rate (MRR) also increases. [6]

5. Steel bridges are vulnerable to corrosion and their surfaces and the process is hazardous to health, so an automatic robotic system would be an alternative. A hexagonal topology based coverage pattern is adopted to reduce unblast areas $\&$ editing process is included to confine blasted areas within boundaries and is verified by simulations based on an industrial robot arm. [7]

6. The erosion rate is PMMA was measured with stream of $25 \mu \mathrm{m} \mathrm{Al}_{2} \mathrm{O}_{3}$; the erosion rate was at $25^{\circ}$. The effect of mask particle interactions was observed. Large discrepancies were seen between the model and the experimental results. [8]

7. The paper describes an algorithm to model a sandblasted glass based on "fog-based height maps". Sand blasted glass is used as a base (canvas) \& the medium of coloring is crayons. The stylized glass paintings generated by algorithm are better suited for non-photorealistic; this paper proposes a new technique of glass painting with different support medium and coloring medium. This paper provides new directions for computer generated glass paintings. [9]

8. In the development of automation controllers the trend has been to move towards soft controllers so as to provide better control, more flexibility and more reliability with intelligent diagnostics of machine faults. So industries have gradually moved from conventional relay logic control to programmable logic control and then to computerized numeric control. The detail study about P.L.C is explained in this paper. [10]

9. Various ways of fabricating channels in silicon are discussed. The various types of silicon etching are explained in this paper. [11]

10. The present paper is an experimental study regarding the influence of abrasive quantity on surface quality generated when low pressures of water jet abrasive cutting are used. [12] 


\section{PURPOSE OF SANDBLASTING:}

\subsection{Significance}

Sandblasting was originally used to sharpen and grind tools. Wood, metal, glass, concrete and brick are some of the substrates that are usable in this process. It will remove any dirt, paint or other substance in preparing the substrate for painting. Sandblasting also refreshes the look of concrete and brick. Sandblast old sidewalks, brick walls, windows and even roofing. Sandblasting can also smooth rough surfaces.

\subsection{Techniques}

There are two types of sandblasting techniques. Wet sandblasting is commonly used for exterior jobs, and dry sandblasting is typically used on interior jobs or special projects. The wet sandblasting process works well to remove paint, stucco and other debris from a substrate.

\subsection{Considerations}

Some cities and towns require a permit for sandblasting. Wet sandblasting is preferable over dry sandblasting as it is less invasive. Before sandblasting, cover structures not needing the process with plastic sheeting to protect them from erosion.

\section{PROBLEM IDENTIFICATION:}

The following problem occurs during glass etching process:

\subsection{Incomplete Reaction with Glass}

The etching chemical may fail to react properly on the glass if the surface has any traces of grime, dust or residue prior to application. You must remove all potential barriers from the glass using water. Avoid using a glass cleaner, as it may leave a film behind. Dry the glass thoroughly after cleaning and avoid putting fingerprints on the etching surface by wearing gloves.

\subsection{Skin Irritation}

The chemicals in the etching substance can burn and irritate the skin.

\subsection{Damage to Sink Enamel}

Over time, rinsing your etching projects off over an enamel or porcelain sink may take its toll on the luster of the basin, especially if you use generous amounts of etching cream or use the product regularly.

\section{AIM AND OBJECTIVE}

Due to the rapid changes in the manufacturing environment, there has become a growing need for computer based systems and automation.

$>$ As the glass etching process is very complicated and it is hazardous to the worker, and also many child labours are involved in etching process and it takes more time. Alternative glass-machining methods are available but they all have certain disadvantages.
$>$ This project is a solution over the limitations of such conventional etching process. The aim to make an automated glass frosting machine at low cost. Because of the powder particles (contamination) and the large minimum feature sizes, the process is carried out with greater flexibility and accurately.

\section{CONCEPT DEVELOPMENT}

\subsection{Action Plan}

The whole work of development is divided into two stages

1. Design and fabrication of Glass frosting machine.

2. Developing a user friendly interface on computer.

\subsubsection{Design and Fabrication Of Glass Frosting Machine}

Before starting the actual fabrication, the study of various types of glasses is done. The material and specification of all components is decided according to size of glass. The assembly modeling and its simulation carried out through Solid works software and fabrication is done. The methodology is represented in pyramid form as shown below in fig 3.1 .

\subsubsection{Developing A User Friendly Interface On Computer}

In the second step of development, a user interface is designed with the help of KIEL software. The event programming (using $\mathrm{C}$ programming language) is done for frosting on glass.

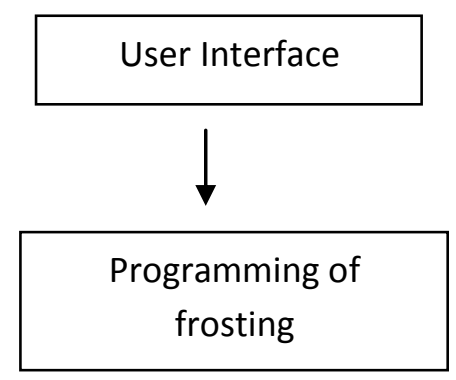

Figure. 1 Developing a User Friendly Interface on Computer

The present invention relates to machine and to certain components, Therefore design for chemically frosting articles made of glass. In spite of increasing demand for such articles, they are still produced on handicraft scale essentially involving manual labor and low productivity. The objective of the proposed project is to make an automated glass frosting machine at low cost. Because of the powder particles (contamination) and the large minimum feature sizes, the process is carried out with greater flexibility and accurately. The aim of the project is to develop a computer assisted glass 
frosting machine with two axes $\mathrm{X} \& \mathrm{Y}$ will be controlled by computer.

Glass frosting involves creating a design on a glass surface by applying an acidic or abrasive substance. Usually found in the form of a cream, etching substances contain caustic chemicals such as ammonium and fluoride as the active ingredients. These are to be handled by adults or completed under adult supervision, as misuse or close contact with etching chemicals may require immediate medical attention.

\subsection{Proposed Concept:}

The box type configuration will be providing the required rigidity and robustness. This type will be beneficial for glass, as the structure have minimum basic configuration, easy for fabrication point of view. It is simplest structures with fewer complications. The working area of glass will be A4 size. Within this area the glass engraving will be done

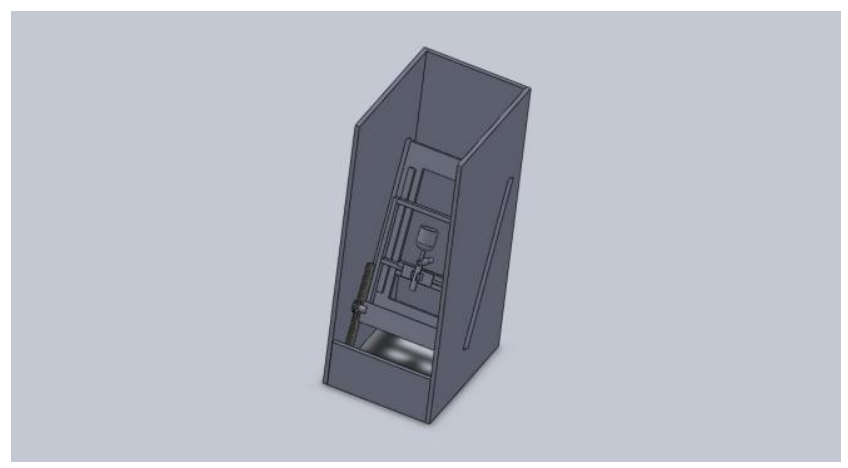

\section{Components:}

Figure 2. Proposed Concept

- Glass

- Sandblasting gun

- Glass mounting frame

- Channels

- Rack and Pinion

- DC. Motor

- Nozzle mounting frame

- Microcontroller

- LCD

- Compressor (6-9 bar)

\section{INTERFACING}

7.1 The complete interface process is done in following sequence.

1. Construction of Hardware by using suitable electronic component according to requirement.

2. Programming for the software interface by using $\mathrm{C}$ language.

3. Integration of above three to get a complete assembly.

\section{2 steps of Programming in C}

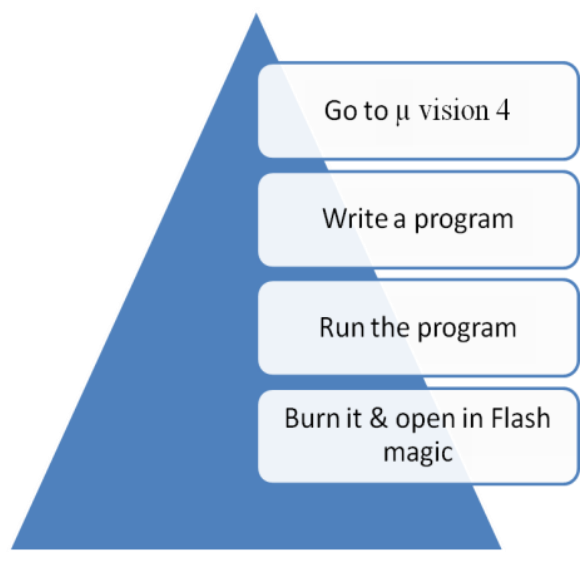

Figure 3. Steps of Programming

\section{RESULTS AND DISCUSSION}

1. It is safer than machine than conventional one because the whole sandblasting is controlled by computer system.

2. 2. As the construction is of box type and door is provided so the operation is performed in cabin so dust will be inside the cabin, it will not affect the worker. As in conventional one the worker needs hand gloves, goggles' etc. for protection.

3. 3. It provides greater accuracy for repetitive work which is quite difficult in conventional one. Conventional requires more attention for getting profiles on various glasses.

4. 4. The most important it increases more productivity and reduced the skilled operator, while in conventional one the skilled worker are involved.

5. The experiment is conducted Successfully with the help of Compressor; also the material removal rate is calculated by using Formula

$$
Q=X Z d^{3} v^{3 / 2}\left\{\frac{\rho}{12 \mathrm{Hw}}\right\}^{3 / 4}
$$


Table No.1 MRR at various Pressures
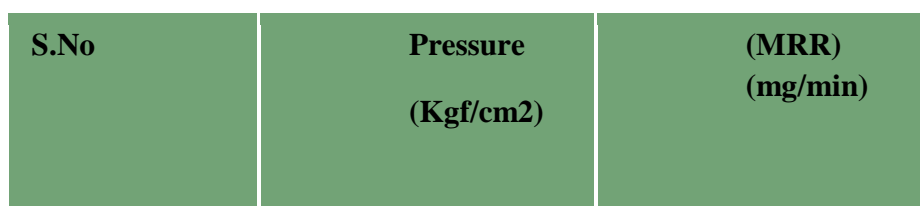

1.

5

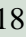

2.

6

3.

7

4.

8
Journal of Advanced Egg. Technology, E-ISSN 0976-3945.

[6] M. Roopa Rani and S. Seshan "Abrasive Jet Machining-Process Variables and Current Application", Metals Materials and Process, 1995 Vol.7 No.4, pp 279-290.

[7] T.R. Ren, N.M. Kwok, D.K. Liu and S.D. Huang, "Path planning for a robot arm sand blasting system", Proceedings of IEEE International conference on Information \& Automation, June 2023,2008 .

[8] H.Getu, A. Ghobeity, J.K. Spelt, M. Papini, "Abrasuve jet micromachining of polymethacrylate", International Journal Elsevier, Wear. 2007.01.063.

[9] Priti Sehgal, P. S Grover, "Stylized Glass Paintings for Non-Photorealistic Rendered scenes" Proceedings of IEEE $9^{\text {th }}$ International Conference on Computer Aided Design and Computer Graphics, 2005.

[10] Pradeep Chatterjee, "An overview of Computerized Numeric Control (C.N.C.) and Programmable Logic Control (P.L.C.) in machine automation", International Journal, 2008.

[11] R. Willem Tjerkstra, Meint de Boer, Erwin Berenschot, J.G.E. Gardeniers, Albert van den Berg, Miko Elwenspoek, "Etching Technology for Microchannels", MESA Research Institute, Netherlands.

[12] Eugen Herghelegiu, Miroslav Radovanovic, Gheorghe Brabie \& Nicolae Catalin Tampu, "Influence of abrasive material quantity on surface Quality generated by abrasive water jet operation" International Journal of Modern Manufacturing Technologies, ISSN 2067-3604, Vol. III, No. 2 / 2011

[1] E. Belloy, A. Sayah, M.A.M. Gijs, "Oblique powder blasting for 3-dimensional micromaching of brittle materials", Swiss Federal Institute of Technology Lausanne, Switzerland, Sens. Actuators A 92 (2001) 358-363.

[2] H. Wensink, J.W. Berenschot, H.V. Jansen, M.C. Elwenspoek, High resolution powder blast micromachining, in: Proceedings of $13^{\text {th }}$ International Conference on Micro Electro Mechanical systems (MEMS-2000), Miyazaki, Japan,23-27 Jan. 2000,pp. 769-774.

[3] Anne-Gabrielle Pawlowski, Eric Belloy, Abdelj alil Sayah, Martin A.M. Gijs, "Powder blasting patterning technology for micro fabrication of complex suspended structures in glass", Swiss Federal Institute of Technology Lausanne, Switzerland, Microelectronic Egg. 67-68 (2003) 557-565.

[4] Hen Wensink, Fabrication of Microstructures by Powder Blasting, Ph.D. Thesis, University of Twente, Netherlands, 2002.

[5] Bhaskar Chandra Kandpal, Naveen Kumar, Rahul Kumar, Rahul Sharma, Sagar Deswal, "Machining of glass and ceramic with alumina and silicon carbide in abrasive jet machining", International 